\title{
EXPLORING DRIVER RESPONSES TO UNEXPECTED AND EXPECTED EVENTS USING PROBABILISTIC TOPIC MODELS
}

\author{
Vindhya Venkatraman ${ }^{* 1,2}$, Yulan Liang${ }^{1}$, Elease J. McLaurin², William J. Horrey ${ }^{1}$, Mary F. \\ Lesch $^{1}$ \\ Liberty Mutual Research Institute for Safety, Hopkinton, Massachusetts, USA ${ }^{1}$ \\ University of Wisconsin-Madison, Madison, Wisconsin, USA ${ }^{2}$ \\ Email: vindhya.venkatraman@libertymutual.com*
}

\begin{abstract}
Summary: Drivers' expectations influence their responses to events in complex ways. In particular, covert and sustained hazards, like crosswinds, might require complex vehicle control adaptations. We investigated differences between drivers' lateral responses in unexpected and expected (repeated) crosswind events using probabilistic topic modeling. First, each driver's event-based steering wheel movements (angle and rate, $5 \mathrm{~Hz}$ ) were transformed into symbolic words. Then, probabilistic topic modeling was used to discover patterns in the steering wheel movement data across the event conditions. Results indicate that drivers may make fewer abrupt steering wheel movements when they encounter unexpected crosswinds. On the contrary, drivers are more likely to make continuous faster steering corrections to compensate crosswinds when they are expected. The topic models also classify unexpected and expected crosswind events better than traditional models that use single aggregated values across events (maximum steering wheel angle and rate). These preliminary insights show an advantage for granular, time-series based analysis of driving data, and suggest a viable machinelearning based technique to conduct such investigations.
\end{abstract}

\section{INTRODUCTION}

Drivers' expectations of events and hazards profoundly affect driving safety. Expectations are products of top-down cognition-learned associations between roadway events and safe vehicle control behaviors - that reflect a driver's readiness to respond to situations, events, and information in predictable and successful ways (Alexander \& Lunenfeld, 1986, p. 8; Lee, 2005). A priori expectations are formed through long-term experience and skill development, whereas ad-hoc expectations develop during a trip based on the evolving road and environment conditions (Alexander \& Lunenfeld, 1986). Both types are assumed to be operative throughout the driving activity, influencing driving tasks such as pre-trip decisions, route planning, attention, detection of hazards, and longitudinal and lateral vehicle control (Lee, 2005).

Substantial research has investigated the effects of expectations on driver reaction times and control maneuvers; however most are focused on discrete roadway events such as decelerating lead vehicles (Green, 2000; Engstrom, Ljung Aust, \& Vistrom, 2010; Lee, McGehee, Brown, \& Reyes, 2002). Repeated exposures to similar events in the same experiment (or trip) might contribute to the development of ad-hoc expectations, and subsequent adaptation of vehicle control. Simulator studies of driver responses to repeated critical lead-vehicle events have found that, in comparison to the first (unexpected) event, drivers release the accelerator pedal faster (Engstrom et al., 2010; Lee et al., 2002) and apply lesser deceleration forces (Lee et al., 2002). 
Steering behaviors, however, have been found to be consistent in both unexpected and repeated events. For example, a study of evasive maneuvers in rear-end collision-imminent events with truck drivers found that maximum steering wheel angles are similar across both unexpected and repeated event exposures (Benderius, Markkula, Wolff, \& Wahde, 2014). The authors also estimated steering wheel reversal rates over the duration of evasive and stabilization maneuvers. These values were similar in both unexpected and repeated events. Maneuvers in discrete events such as collision avoidance may be qualitatively different from sustained vehicle control in events such as crosswind gusts. These differences might arise because continuous steering responses and traditional measures such as averages of responses over the event duration or maximum values might not capture the behavior sufficiently. A granular analysis that considers the temporal evolution of drivers' lateral and longitudinal responses at smaller timescales may help understand how drivers progressively counter the effects of sustained hazards such as crosswinds. Unlike traditional data analysis methods (e.g. ANOVA), machine learning approaches afford opportunities to perform granular analysis, discover unknown patterns in the data, and link patterns to probable real-world meanings. Machine learning approaches often do not require rigorous assumptions, and methods used for one data type can be suitably modified for another data type. For example, one study applied topic modeling - a natural language processing method - to numeric driving data and differentiated driving patterns between drivers with obstructive sleep apnea and normal drivers (McLaurin, McDonald, Lee, Aksan, Dawson, Tippin \& Rizzo, 2014).

Probabilistic topic modeling is an unsupervised machine learning method that was originally used to search for topics in a collection of text documents (Blei, 2012). Each document is viewed as a mixture of a number of topics of varying importance. A metaphor for this idea is the set of keywords presented for an academic journal article. The importance of each topic is manifest by its frequency in a given document. Topics are defined by groups of words that co-occur frequently. The output of the topic modeling analysis include topics, the probabilistic distributions of words across different topics (i.e., what words define each topic), and the probabilistic distributions of all topics in each document. When applied to numeric driving data, the symbolic aggregate approximation (SAX) algorithm (Lin, Keogh, Wei, \& Lonardi, 2007) can be used to convert numeric data into a symbolic form.

In this study, we sought to extract meaningful patterns in drivers' lateral vehicle control during unexpected and expected crosswind events using probabilistic topic modeling. Understanding the evolution of drivers' vehicle control over exposure to environmental events (e.g. crosswinds) could aid development of driver models for use in vehicle safety technologies such as crosswind assists, lane-centering technologies, and electronic stability control systems.

\section{METHODS}

\section{Data Source: Simulator Study}

Data from two simulator experiments were used in this analysis. The experiments were part of a series of studies that examined drivers' subjective appraisals of their own driving performance. A fixed-base driving simulator with an open cab configuration was used in both experiments. The two experiments varied in terms of the post-trial subjective ratings employed (Horrey, Lesch, \& 
Liang, 2016), but for the purposes of the current analysis, these differences are assumed to be inconsequential. All other aspects of the experimental trials were identical across the studies. A total of 41 participants were recruited-20 in Experiment 1 (gender balanced, $M=39.4$ years, $\mathrm{SD}=11.9)$ and 21 in Experiment 2 (10 males, 11 females, $\mathrm{M}=37.2$ years, $\mathrm{SD}=12.1)$. All participants possessed a valid drivers' license. Each experiment consisted of eight driving blocks, each lasting approximately 10 minutes. All the driving blocks required drivers to drive on straight rural highways under varying levels of traffic (low and high density). Within each block, eighteen 25-second trials were presented, interspersed with normal driving. The trials varied by two levels of lateral crosswind. The high wind gusts were between $1000-1300 \mathrm{~N}$, and the low wind gusts were between $500-800 \mathrm{~N}$. The lateral direction of each wind gust (left or right) was random. Thus each driver experienced nine trials with low wind gusts and nine trials with high wind gusts in each driving block; the order of presentation was random. Throughout the drives, the drivers' lateral vehicle control (i.e., steering wheel movements) was recorded.

\section{Data Preparation}

In this study, we focused on driver steering responses (lateral control) to the conditions of high wind gusts. Only data from the first driving block for each participant in each experiment was used; this was to minimize confounding effects due to increasing fatigue from repeated exposures. In the first experimental drive, we defined the first trial of high wind gusts as the unexpected event and the last trial of high wind gusts as the expected event. It was assumed that the drivers had developed ad-hoc expectations of the control maneuvers to counter the wind gust before the expected trial, because drivers had experienced eight high wind gust trials within a short time period (i.e., 7-8 minutes). We did not account for the effect of traffic density in the current analysis as no main effect of traffic density was found on the standard deviation of lane position (Horrey et al., 2016).

Steering wheel angle and the rate of change of steering wheel angle were the two lateral control behavior measures used in the probabilistic topic models. Steering wheel angle was calculated as the absolute value of the steering wheel angle in degrees, with the 0 -angle corresponding to the steering wheel resting position. The rate of change of steering wheel angle described how quickly drivers rotated the steering wheel, and was calculated as the absolute value of the degrees of rotation per second. The absolute values were used to maximize interpretation of the magnitude of drivers' responses, given that the wind gusts were administered in either cross lateral direction.

To build the topic models, the numeric driving data was first converted to text data by aggregating the values (steering wheel angle and rate) over a 200-millisecond time window. Then these aggregated values were transformed into symbolic words and documents using SAX. The SAX method divides each measure into four equally-likely bins using percentiles. That is, the data was binned into $0-25^{\text {th }}, 26^{\text {th }}-50^{\text {th }}, 51^{\text {th }}-75^{\text {th }}$, and $76^{\text {th }}-100^{\text {th }}$ percentiles of the variables. Because the distributions of steering wheel angle and rate were not normally distributed, we used the log values to determine the bins (Table 1). After that, we assigned a symbol (alphabet) for each bin. For each 200-millisecond time window, we combined the symbols representing the values of the two variables to form a word. The collection of such symbolic words for either the $1^{\text {st }}$ trial or the last trial of high wind gusts of a participant was considered as a document- 
unexpected trial document or expected trial document. Therefore, we had 82 documents in total (two documents for each of 41 participants) and approximately 125 words in each document.

Table 1. Transformation of numeric variables into symbolic form

\begin{tabular}{|c|c|c|c|c|}
\hline \multicolumn{2}{|c|}{ Thresholds to create bins } & \multicolumn{3}{|c|}{ Transform numeric values into a word } \\
\hline \multirow{2}{*}{$\begin{array}{l}\text { Steering Angle in deg } \\
\text { (log-transformed) }\end{array}$} & \multirow{2}{*}{$\begin{array}{c}\text { Steering Angle Rate in deg/s } \\
\text { (log-transformed) }\end{array}$} & \multicolumn{2}{|c|}{ Numeric variable } & \multirow{2}{*}{$\frac{\text { Symbolic form }}{\text { word }}$} \\
\hline & & $\begin{array}{l}\text { Log } \\
\text { (steer) }\end{array}$ & $\begin{array}{l}\text { Log } \\
\text { (steerRate) }\end{array}$ & \\
\hline$-025 \sim 057 \cdot$ smalldeo & $032 \sim 118$ smallrate & 0.61 & 1.51 & meddeg medrate \\
\hline 0.57 1.11: meddeg & $1.18 \sim 1.86:$ medrate & 0.31 & 0.25 & smalldeg_norate \\
\hline largedeg & largerate & $\ldots$ & $\ldots$ & $\ldots$ \\
\hline
\end{tabular}

\section{RESULTS}

The analysis was conducted using the $\mathrm{R}$ statistical analysis software, version 3.3.1 (R Core Team, 2016). We merged all the unexpected and expected trial documents together. The stm package (Roberts, Stewart, \& Tingley, 2016) was used for the development of the topic models and the number of topics was preset to four (lowest residual dispersion). The four topics are illustrated as word clouds; one cloud for each topic (Figure 1).

Topic 1

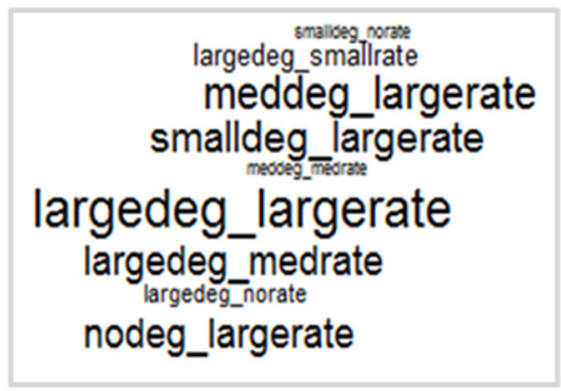

Topic 3

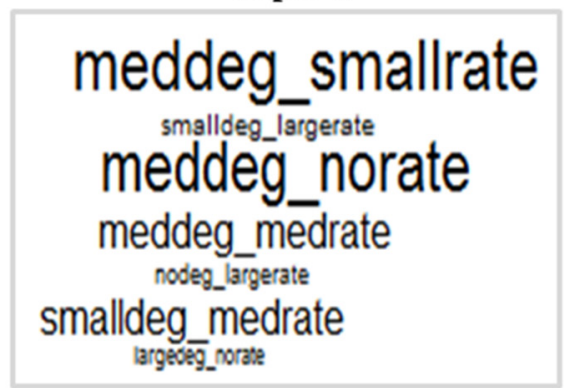

Topic 2

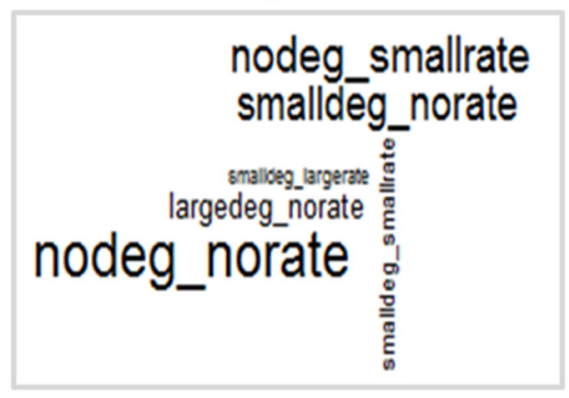

Topic 4

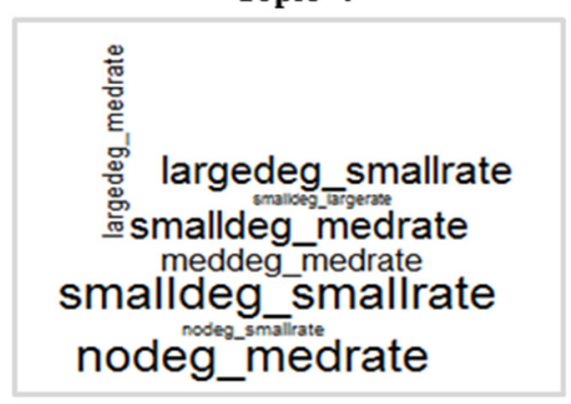

Figure 1. Word clouds for four topics

The size of a word in the cloud indicates the word frequency within a particular topic (Figure 1). Hence, larger words indicate that the word has greater importance in the topic. In Topic 1, the majority of words describe maneuvers of large rate of change of steering wheel angle for any absolute steering wheel angle (large, median, low, or no). Note that the "nodeg" and "norate" indicate negligible values of steering wheel angle and rate. For a large value of steering wheel 
angle, the frequency of words gradually decreased as the steering rate decreased. Topic 2 focused on negligible (or slower) steering wheel rate; the frequent and important words include "nodeg_norate", "smalldeg_norate", "nodeg_smallrate", and "largedeg_norate". Topic 3 was associated with medium values of steering wheel angle including negligible, small, or medium movement rates. Topic 4 describes small to medium changes in steering wheel angle rates. The distribution of words across topics are illustrated (Figure 2).

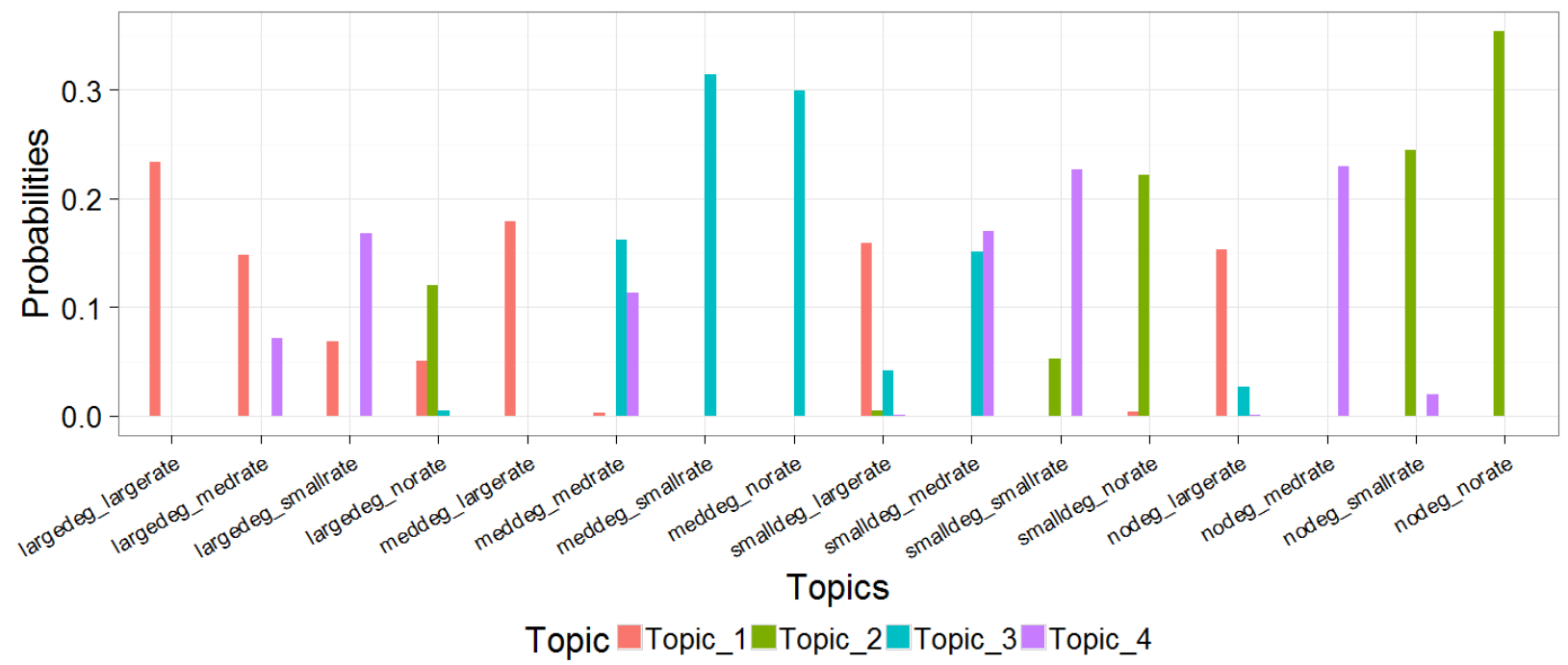

Figure 2. Distribution of word probabilities across topics

These distinct differences between Topics 1 and 2 resulted in slightly different distributions for the expected events and unexpected events (Figure 3) although with large variances. Expected events had higher proportion of Topic 1, and unexpected events had higher proportion of Topic 2. Topic 3 and Topic 4 would not distinguish between expected and unexpected events (Figure 3). Topic 3 was associated with medium values of steering wheel angle including negligible, small, or medium movement rates. Topic 4 focuses on small to medium changes in steering wheel angle rates. The distribution of words across topics are illustrated (Figure 3 ).

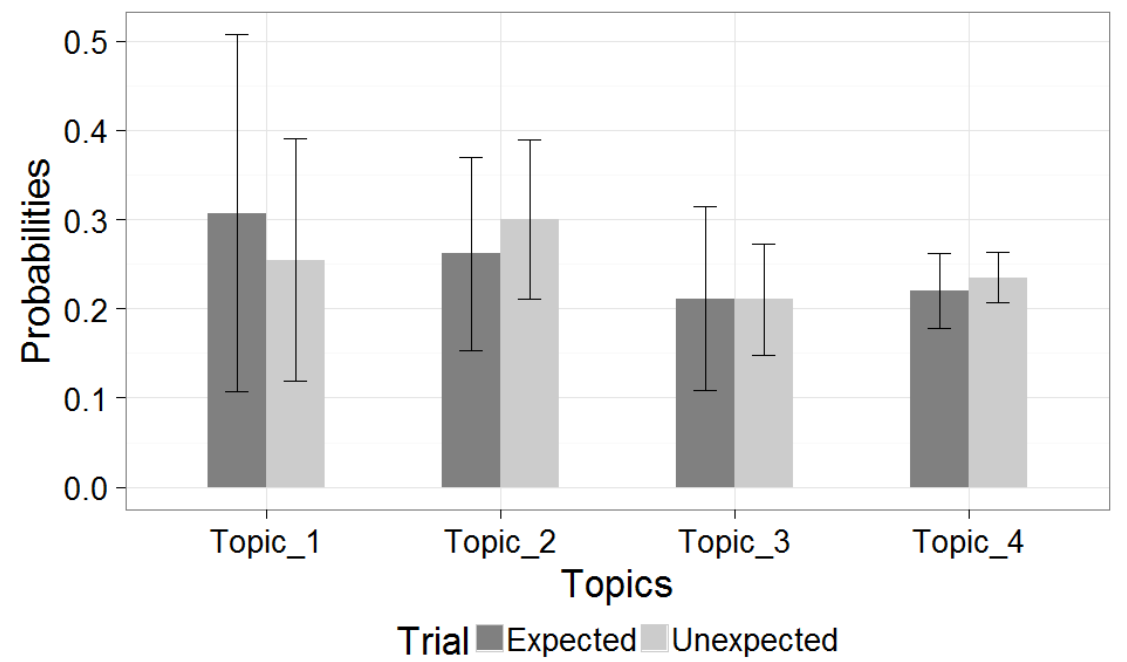

Figure 3. Distributions of topic probabilities between expected and unexpected trials 


\section{Predictive Models}

We built a Random Forest model (Breiman, 2001) to predict whether a given driving trial was an unexpected or expected event using the proportion of the topics for that trial as predictors. We also calculated the maximum steering angle and rate over the duration of the different trials and used these values to build another Random Forest model; this represents the traditional approach of estimating event-based averages for driving data.

The predictive capabilities of the two approaches (topic models and traditional measures) were compared using their Receiver Operating Characteristic (ROC) curves (Figure 4). The topic models perform slightly better $(\mathrm{AUC}=0.67)$ in predicting if a given trial was unexpected or expected event than the traditional models $(\mathrm{AUC}=0.6)$.

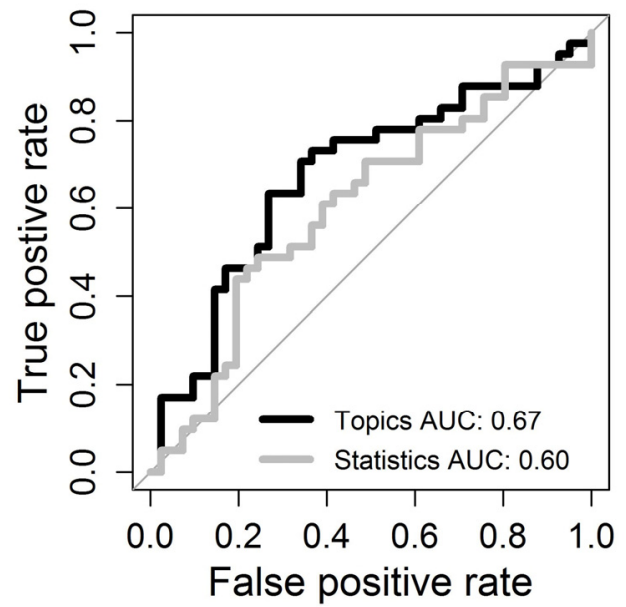

Figure 4. ROC curve comparison between topic model (black line) and traditional statistical model (gray line)

\section{DISCUSSION}

Driver expectations may influence driver responses to covert, sustained hazards in qualitatively different ways than discrete hazards. In this study, probabilistic topic modeling was used to explore differences between drivers' steering maneuvers in ad-hoc unexpected and expected crosswind events (Figures 1, 2, and 3). Resulting topics were applied in a random forest model and provided slightly better classification of driver expectations than a traditional model (Figure 4). Overall, results show that probabilistic topic modeling may be useful in extracting patterns in driving data. More broadly, as shown by this analysis, topic models and machine learning methods provide a viable means to explore factors that span many timescales (e.g. expectations, behavioral adaptation, and skill degradation).

The preliminary qualitative insights from this study motivate further exploration for purposes including driver model development and vehicle technology sign. For example, two of four topics-Topic 1 and Topic 2-provided insights into lateral control behaviors that might otherwise be difficult to obtain. Topic 1, with large steering rates, occurs in greater proportion in expected events. Drivers are likely making continuous steering corrections and reach their intended steering angles faster. In other words they have adapted to the demands of the situation. In the unexpected events, Topic 2 occurs in slightly greater proportion than Topic 1 (Figure 2). More of the steering wheel movement are hence smaller corrections to angle and rate (see Figure 3 ). When the larger movements are made in the unexpected events, they are abrupt, indicating that drivers are starting to adapt responses to the crosswind disturbances.

\section{ACKNOWLEDGEMENTS}

The authors are thankful to three anonymous conference reviewers, and Dr. Yueng-hsiang Huang and Dr. Marvin Dainoff at the Liberty Mutual Research Institute for Safety (LMRIS) for reviews of previous drafts of this paper. We are also grateful to Angela Garabet, Lucinda Simmons, 
Sarah Hong, and Susan Jeffries at LMRIS for their help with data collection and technical assistance.

\section{REFERENCES}

Alexander, G. J., \& Lunenfeld, H. (1986). Driver expectancy in highway design and traffic operations. US Department of Transportation, Federal Highway Administration, Office of Traffic Operations.

Blei, D. M. (2012). Probabilistic topic models. Communications of the ACM, 55(4), 77. doi:10.1145/2133806.2133826

Breiman, L. Random forests. Machine Learning, 45(1): 5-32, 2001. 18

Engström, J., Aust, M. L., \& Viström, M. (2010). Effects of working memory load and repeated scenario exposure on emergency braking performance. Human Factors: The Journal of the Human Factors and Ergonomics Society, 52(5), 551-559.

Green, M. (2000). "How Long Does It Take to Stop?" Methodological Analysis of Driver Perception-Brake Times. Transportation human factors, 2(3), 195-216.

Horrey, W. J., Lesch, M. F., \& Liang, Y. (2016, September). Drivers' calibration in selfevaluated performance: The role of task-related workload and scale specificity. In Proceedings of the Human Factors and Ergonomics Society Annual Meeting (Vol. 60, No. 1, pp. 1904-1904). SAGE Publications.

Roberts, M. E., Stewart, B. M., and Tingley, D. (2016). stm: R Package for Structural Topic Models. http://www.structuraltopicmodel.com

Lee, J. D., McGehee, D. V., Brown, T. L., \& Reyes, M. L. (2002). Collision warning timing, driver distraction, and driver response to imminent rear-end collisions in a high-fidelity driving simulator. Human Factors: The Journal of the Human Factors and Ergonomics Society, 44(2), 314-334.

Lee, J. D. (2005). Driving safety. Reviews of human factors and ergonomics, 1(1), 172-218.

Lin, J., Keogh, E., Wei, L., \& Lonardi, S. (2007). Experiencing SAX: a novel symbolic representation of time series. Data Mining and Knowledge Discovery, 15(2), 107-144. doi:10.1007/s10618-007-0064-z.

McLaurin, E., McDonald, A. D., Lee, J. D., Aksan, N., Dawson, J., Tippin, J., \& Rizzo, M. (2014, September). Variations on a theme Topic modeling of naturalistic driving data. In Proceedings of the Human Factors and Ergonomics Society Annual Meeting (Vol. 58, No. 1, pp. 2107-2111). Sage Publications.

R Core Team (2016). R: A language and environment for statistical computing. R Foundation for Statistical Computing, Vienna, Austria. https://www.R-project.org/. 